\title{
An XYZ Micromanipulator with three translational degrees of freedom
}

Kimberly A. Jensen

Craig P. Lusk

Larry L. Howell

Ihowell@byu.edu

Follow this and additional works at: https://scholarsarchive.byu.edu/facpub

Part of the Mechanical Engineering Commons

Original Publication Citation

Robotica 24.3(May 26): 35-314.

\section{BYU ScholarsArchive Citation}

Jensen, Kimberly A.; Lusk, Craig P.; and Howell, Larry L., "An XYZ Micromanipulator with three translational degrees of freedom" (2006). Faculty Publications. 313.

https://scholarsarchive.byu.edu/facpub/313

This Peer-Reviewed Article is brought to you for free and open access by BYU ScholarsArchive. It has been accepted for inclusion in Faculty Publications by an authorized administrator of BYU ScholarsArchive. For more information, please contact ellen_amatangelo@byu.edu. 


\section{An XYZ Micromanipulator with three translational degrees of freedom \\ Kimberly A. Jensen, Craig P. Lusk and Larry L. Howell*}

Department of Mechanical Engineering, Brigham Young University, Provo, UT 84602 (USA)

(Received in Final Form: June 14, 2005, first published online 21 February 2006)

\section{SUMMARY}

This paper introduces a three degree of freedom $\mathrm{XYZ}$ Micromanipulator (XYZM) that is fabricated in the $x-y$ plane and positions components in the $x, y$, and $z$ directions using three independent linear inputs. The mechanism positions components on a platform using three legs, each composed of a slider mechanism and a parallelogram mechanism.

Three versions of the XYZM were fabricated and tested using surface micromachining processes: the rigid-body, offset, and compliant XYZM. Slider displacements of 45 micrometers result in a predicted out-of-plane displacement of 188 micrometers for the rigid-body XYZM, 205 micrometers for the offset XYZM, and 262 micrometers for the compliant XYZM.

KEYWORDS: XYZ Micromanipulator; Three inputs; Translational DOFs.

\section{INTRODUCTION}

Micropositioning through the use of microelectromechanical systems (MEMS) has been demonstrated through the positioning of fibers, ${ }^{1}$ lenses,${ }^{2-5}$ mirrors, ${ }^{6,7}$ shutters,,${ }^{8,9}$ and gratings. ${ }^{10}$ This paper introduces a three degree of freedom micropositioning mechanism that positions microcomponents in the $x, y$, and $z$ directions. The device is fabricated in-plane and allows the positioning of components out-of-plane using three independent linear inputs. The positioning platform of the mechanism remains horizontal throughout the motion of the device due to the parallelguiding mechanisms in each leg.

The results of this research allows the development of alternative methods for moving lenses, gratings, and other components as well as further development of ortho-planar MEMS with out-of-plane displacements over 50 micrometers. This device also allows for the positioning of microcomponents in the $x, y$, and $z$ directions by using only three linear inputs instead of four or six.

\section{XYZ MICROMANIPULATOR (XYZM)}

Tsai and Stamper ${ }^{11}$ and Tsai ${ }^{12}$ developed a parallel manipulator with three translational degrees of freedom where the moving platform is constrained to a translational motion.

\footnotetext{
* Corresponding author: E-mail: lhowell@byu.edu
}

The special motion was achieved by using 17 links and 21 revolute joints to create 3 identical limbs consisting of a parallelogram mechanism and link, connected to the fixed base and moving platform by revolute joints. Although this is a macro device constructed of traditional macro components, was not designed to be planar, and used rotational inputs at joints, it still provided a valuable starting point in developing the XYZM. Other macro three DOF translational parallel manipulators are described in reference [13].

The challenges of the microregime, such as planar fabrication, stiction, mechanism manipulation, and force magnitudes, govern the design and method of actuation of the XYZM. MEMS are fabricated using surface micromachining methods which are similar to those used in the manufacture of integrated circuits.

In the surface micromachining process, thin films of polysilicon and silicon dioxide are deposited on a single crystal silicon substrate and etched to create freestanding devices. Polysilicon is generally deposited on the substrate using Low Pressure Chemical Vapor Deposition (LPCVD) while the oxide layers are grown by thermal oxidation. Generally, these thin films, or layers, are one to two micrometers thick. For a MEMS device, the polysilicon films serve as structural layers while the oxide films form the sacrificial layers. Following the deposition of a silicon nitride layer on the substrate, the structural and sacrificial layers are deposited, alternatively, with each layer being patterned using photolithography. In photolithography, photoresist, a light sensitive polymer, is deposited on the thin films according to the desired layer geometry. The area is then exposed to UV light which changes the properties of the thin film and allows the areas not covered by photoresist to be rinsed away. By photolithography, the length and width of each layer can be controlled. Following this sequence of depositing and patterning each layer, the structural layers are "released" by dissolving the sacrificial layers using hydroflouric acid. The structural layers form the mechanism. The surface micromachining process can be seen in Figure 1. Two surface micromachining processes were used in this research - the MultiUser MEMS Processes (MUMPs) and the Sandia Ultraplanar Multi-level MEMS Technology V (SUMMiT V). Often, as layers are deposited, the layers conform to previous layers underneath. Because of this conformity and the number of layers available in the fabrication process, the rigid links, hinges, and joints in the XYZM were designed and fabricated on the same plane, as illustrated by Figure 2. 

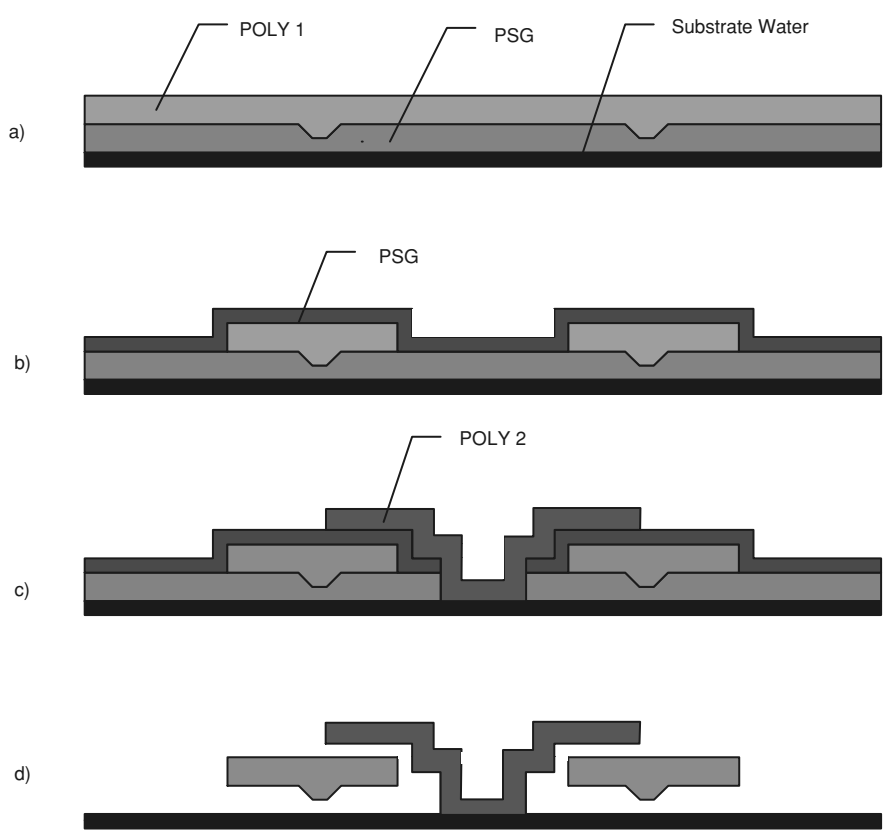

Fig. 1. The Surface Micromachining Process in MUMPS a) the deposition of the sacrificial PSG layer and Poly $1 \mathrm{~b}$ ) after Poly 1 has been patterned, the deposition of the second sacrificial PSG layer c) Poly 2 deposited and patterned d) the "release" of the structural layers.

Actuation on the microlevel was achieved by introducing prismatic joints into the design. The lower links of each individual leg are controlled by sliders which allow the device to be controlled with three independent inputs in the $x y$ plane.

This micropositioning mechanism is composed of a circular positioning platform with three legs equally spaced around the platform. Each leg is composed of two sections: a slider mechanism and a parallelogram mechanism. Each section is connected to another section by a micro hinge.

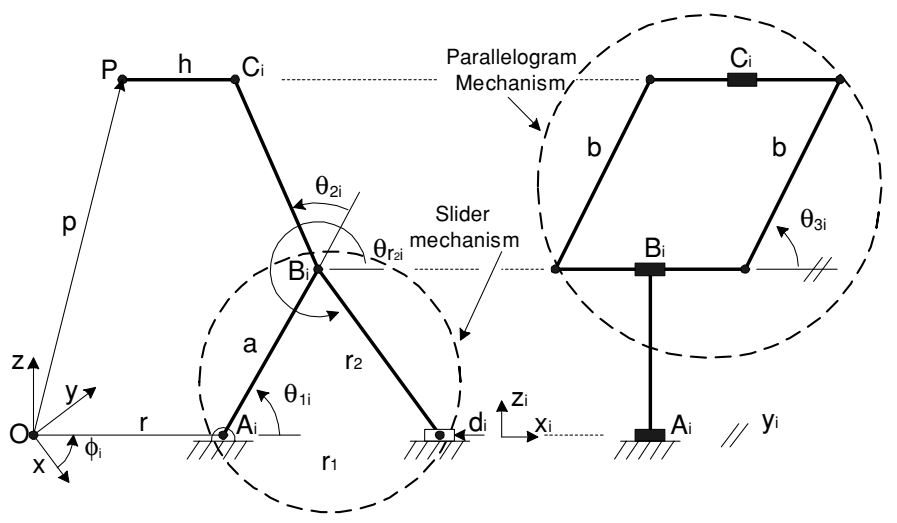

a)

b)

Fig. 3. Diagram of one leg of the 3 DOF XYZ micromanipulator with parameters labeled. a) A side view of the leg and b) shows the front view of the leg.

Pin joints are used in the parallelogram mechanism to allow rotation. The top of the parallel mechanism is connected to the positioning platform through a microhinge. Figure 2 shows a schematic for the top view of the XYZM. Figure 3 shows the front and side view of a single leg of the XYZM and illustrates the different sections of each leg and their connectivity. A rendering of the XYZM in a raised position is illustrated in Figure 4.

In addition to the XYZM, an offset XYZM (OXYZM) was fabricated to simplify fabrication using the MUMPs processes. In the OXYZM, the parallelogram mechanism is offset from the microhinges at $B_{i}$ and $C_{i}$, by $f_{1}$ and $f_{2}$, to allow room for floating pin joints, as shown in Figure 5. The front and side view of a single leg of the OXYZM is shown in Figure 6.

The design of the legs allows the entire mechanism to have a total of three degrees of freedom. The parallelogram

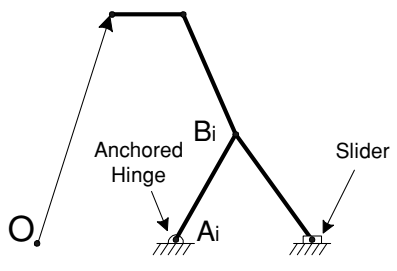

a)

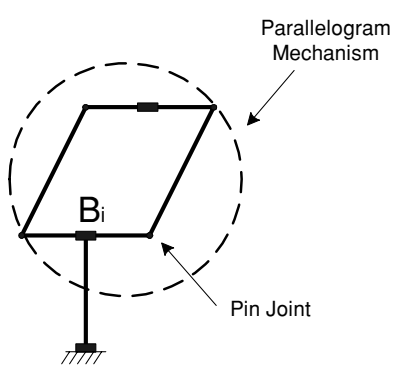

b)

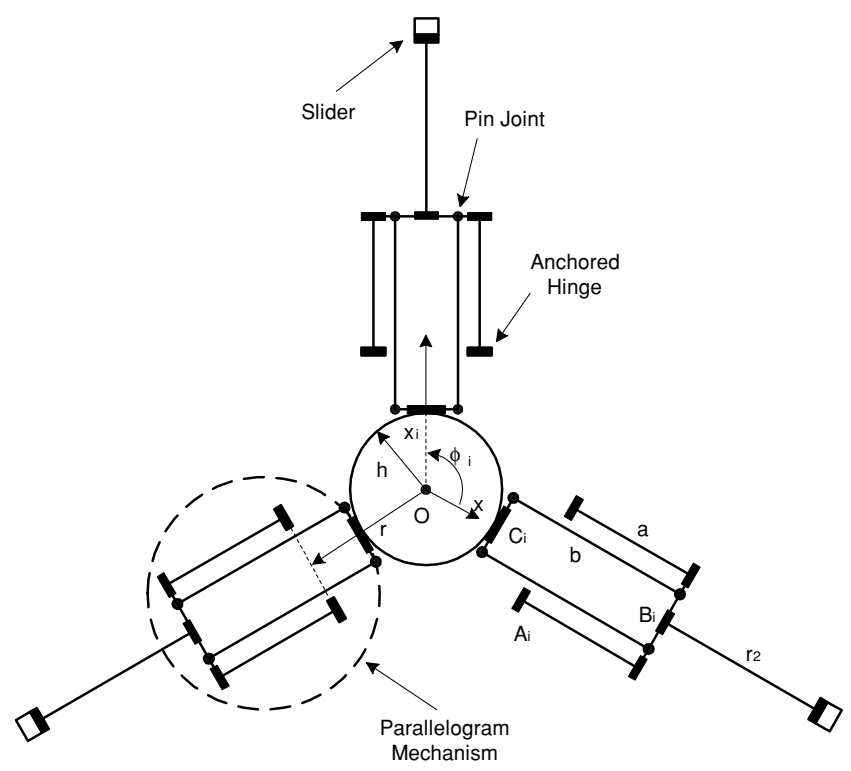

c)

Fig. 2. Schematic of three degree of freedom XYZ micromanipulator. a) Side view of a single leg. b) front view of parallelmechanism in single leg that allows $\mathrm{X}-\mathrm{y}$ positioning of platform. and c) top view of entire XYZ micromanipulator. 


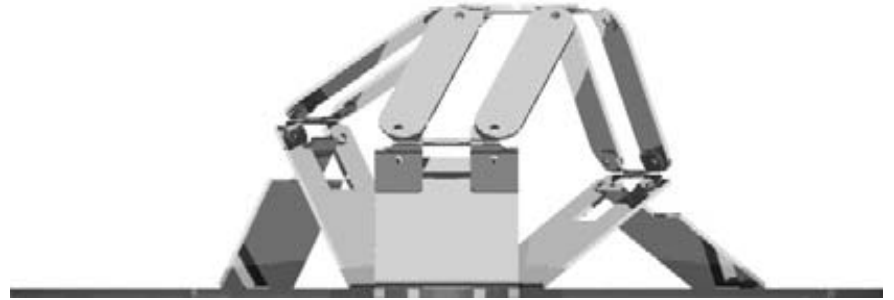

Fig. 4. A rendering of the $\mathrm{XYZ}$ micromanipulator in a raised position.

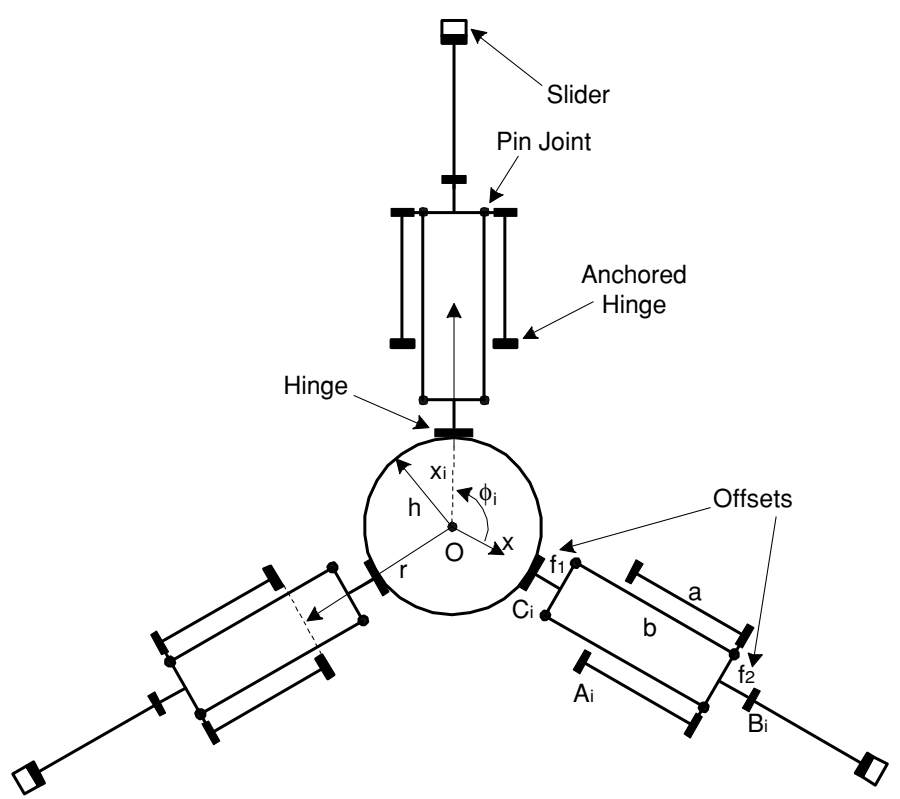

Fig. 5. Schematic of top view for offset three degree of freedom $\mathrm{XYZ}$ micromanipulator.

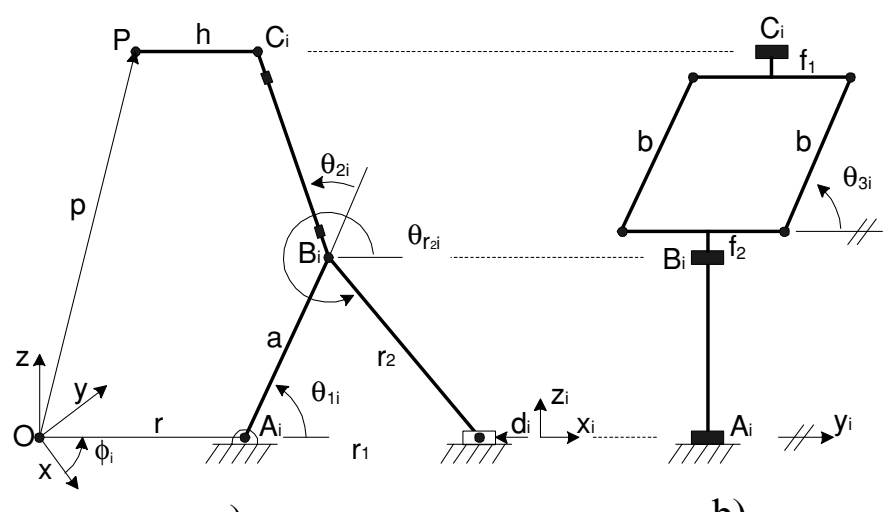

a)

b)

Fig. 6. Diagram of one leg of offset XYZM. a) A side view of the leg and $b$ ) shows the front view of the leg.

mechanism is a 4-bar mechanism where opposing links remain parallel throughout its motion. It was used in the top section of each leg to reduce the number of degrees of freedom, thus eliminating rotation in the top link and allowing the platform to remain horizontal throughout the device's motion. The slider mechanism provides the input to the mechanism and allows the out-of-plane motion of the device. The position of the platform is ultimately dependent on the three independent input displacements of the slider of each leg.

Ortho-planar mechanisms are mechanisms that can have all links simultaneously located in the same plane with motion occurring out of that plane. ${ }^{14}$ Generally, orthoplanar mechanisms can go into more than one configuration. Because MEMS are fabricated in plane, orthoplanar mechanisms generally result when motion of the device occurs out-of-plane.

\section{FABRICATION OF THE XYZ MICROMANIPULATOR}

The XYZ Micromanipulator (XYZM) was fabricated using two surface micromachining processes: the MultiUser MEMS Process (MUMPs) and Sandia's Ultra-planar Multilevel MEMS Technology V (SUMMiT V).

The MUMPs process uses two structural conformal layers of polycrystalline silicon (polysilicon). ${ }^{15}$ SUMMiT V uses four structural layers. ${ }^{16}$ In SUMMiT V, some of the sacrificial layers are planarized using chemical mechanical polishing, therefore eliminating conformity in the third and fourth polysilicon layers. These layers of polysilicon were used to create a mechanism composed of pin joints, hinges, sliders, rails, and a positioning platform. Figure 7 shows a single leg of the compliant XYZ Micromanipulator.

Limitations in the surface micromachining process govern the placement and geometry of the legs. In MUMPs, the legs were fabricated as illustrated in Figure 5. From this schematic, it is seen that links are separated and not layered over each other to eliminate conformity problems. Since conformity can be avoided in SUMMiT V, the prototypes were fabricated with the lower link of the leg over the slider mechanism. This layering approach reduces the overall footprint of the device. Figure 8 shows the XYZM fabricated in SUMMiT V.

\section{III.1. Pin joints}

For planar rotation, such as with the parallelogram mechanism in the upper segment of each leg, pin joints

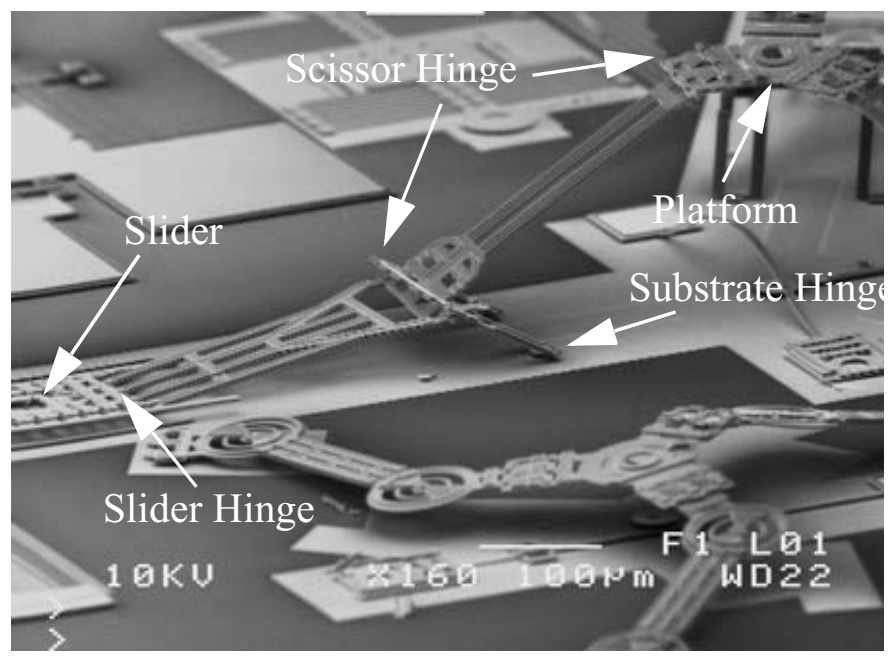

Fig. 7. SEM of single leg of Compliant XYZ Micromanipulator with components labeled. 


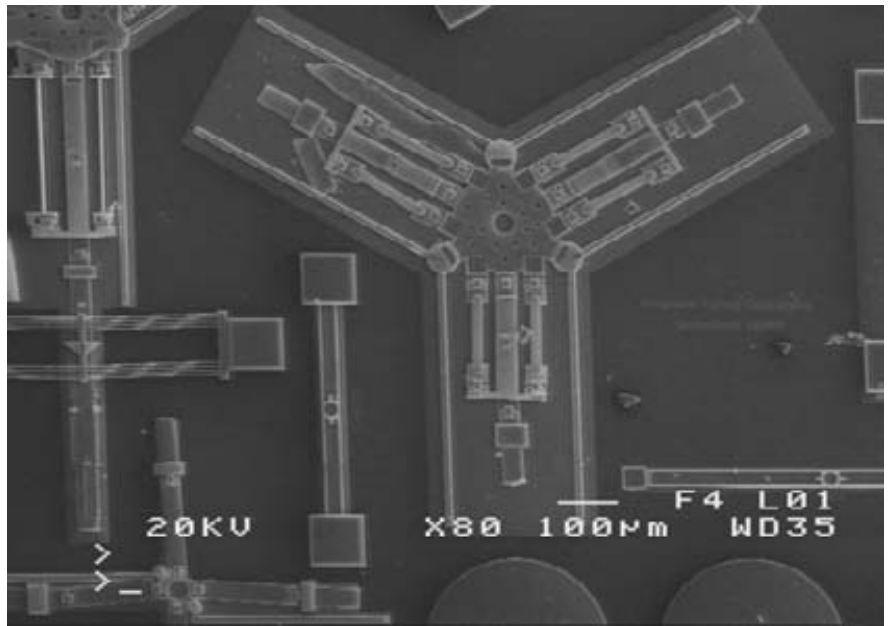

Fig. 8. SEM of XYZ Micromanipulator fabricated in SUMMi T V.

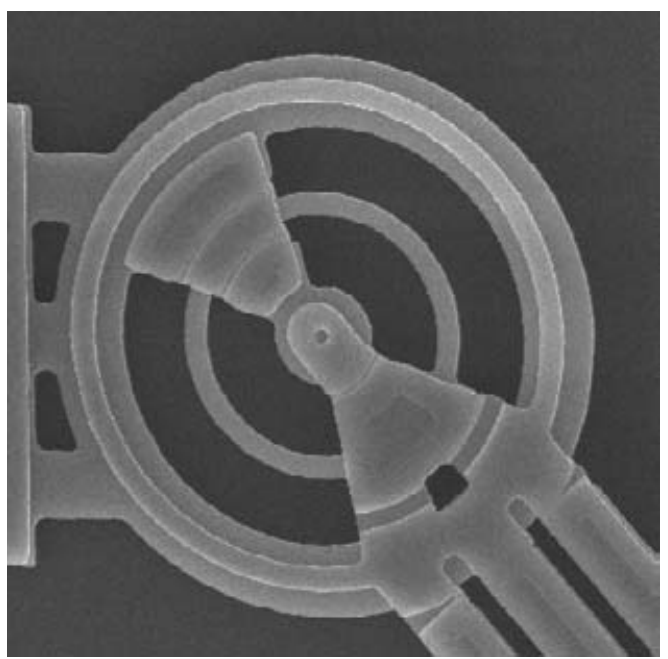

Fig. 9. SEM of a pin joint used in rigidbody XYZMM.

were used and fabricated using two layers of polysilicon. Polysilicon was used to fabricate a pin and hub. In the positioning mechanism, the first polysilicon layer was used to create the pin of the joint, while the second polysilicon layer was used to create the hub. Figure 9 shows a pin joint used in the XYZM fabricated in MUMPs. ${ }^{17}$

\section{III.2. Microhinges}

The XYZM uses four types of microhinges that allow the mechanism to rotate out-of-plane: the substrate or anchored hinge, the scissor hinge, the slider hinge, and the released hinge.

\section{III.3. Substrate hinge}

The substrate hinge is made from two layers of polysilicon and is composed of a hinge pin and a confining "staple". ${ }^{18-20}$ The axle of the hinge is connected to the rotating member of the device.

The axle is fabricated using one layer of polysilicon. The second polysilicon layer is then deposited over the axle creating the "staple" or bridge. This staple is anchored to the substrate, allowing the first polysilicon layer to rotate

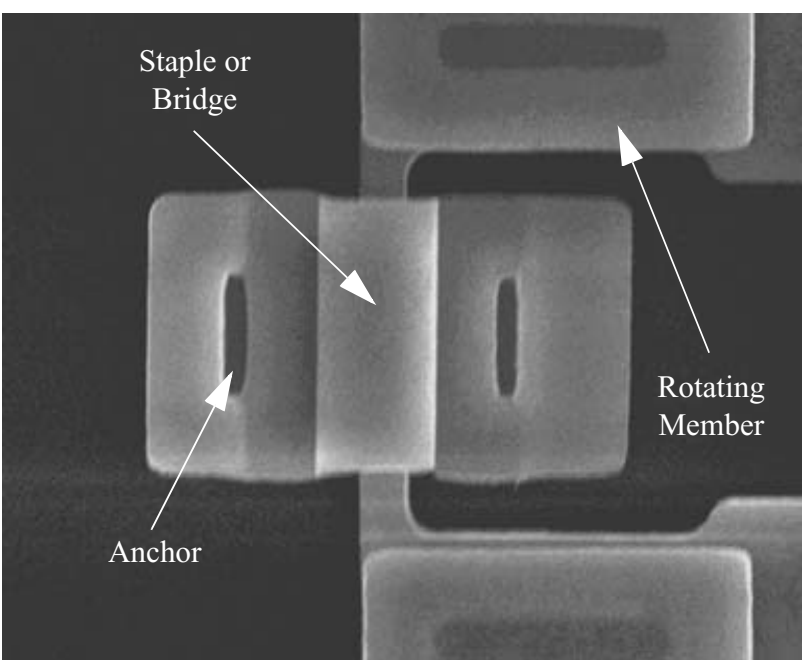

Fig. 10. SEM of a substrate hinge.

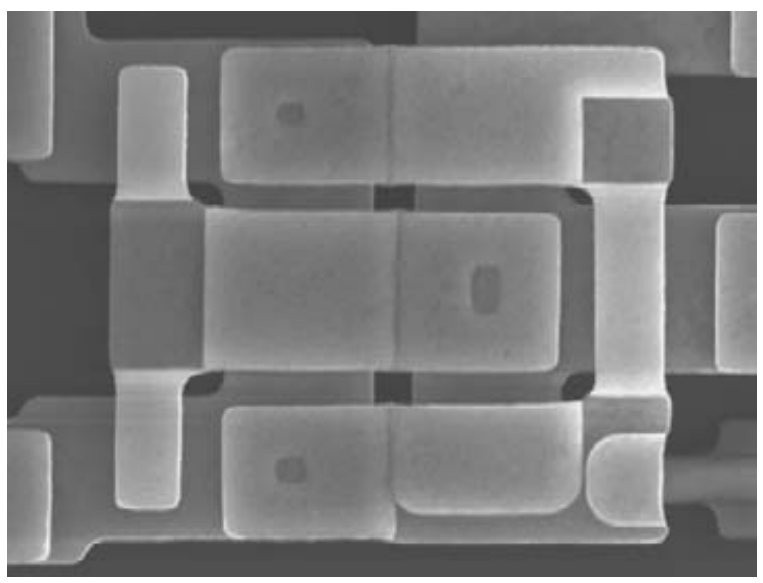

Fig. 11. SEM of a scissor hinge.

under the second polysilicon layer. An SEM of a substrate hinge used in the XYZM is shown in Figure 10.

\section{III.4. Scissor hinge}

Scissor hinges are not connected to the substrate and connect two released members of the mechanism. ${ }^{18,19}$ Instead of employing an anchored staple, scissor hinges interlace two polysilicon parts to allow rotation of a component. With the scissor hinge, the member can only rotate 180 degrees. One polysilicon part forms the rotating axle and plate. The other polysilicon part is then layered under and over this axle pin. The interweaving is accomplished by connecting the first and second polysilicon layers at different points in the hinge. Figure 11 shows the scissor hinge of the XYZM and the interlacing of the polysilicon layers.

\section{III.5. Slider hinge}

The slider hinge is similar to the substrate hinge, except the confining staple in the slider hinge is not fixed to the substrate, ${ }^{19}$ but is fixed to the first released polysilicon layer, allowing the hinge to slide across the substrate. The slider remains in contact with the substrate because it is confined 


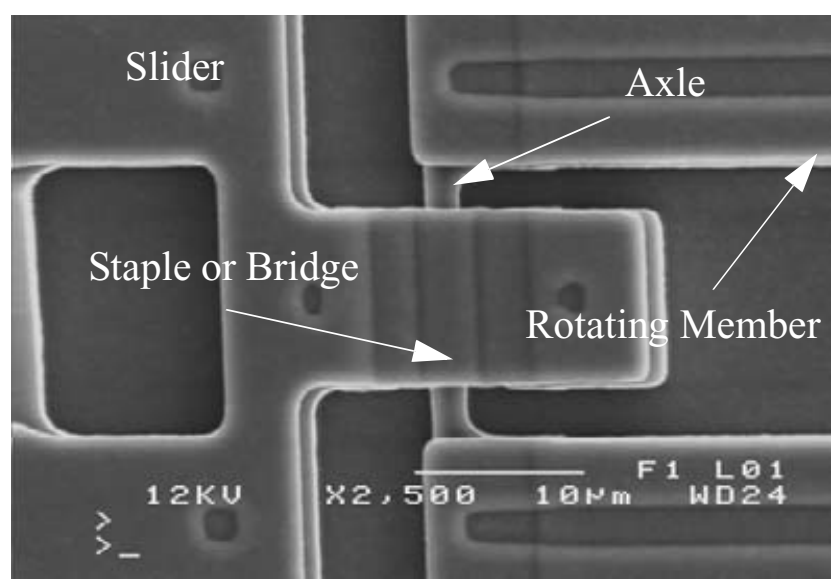

Fig. 12. SEM of a slider hinge.

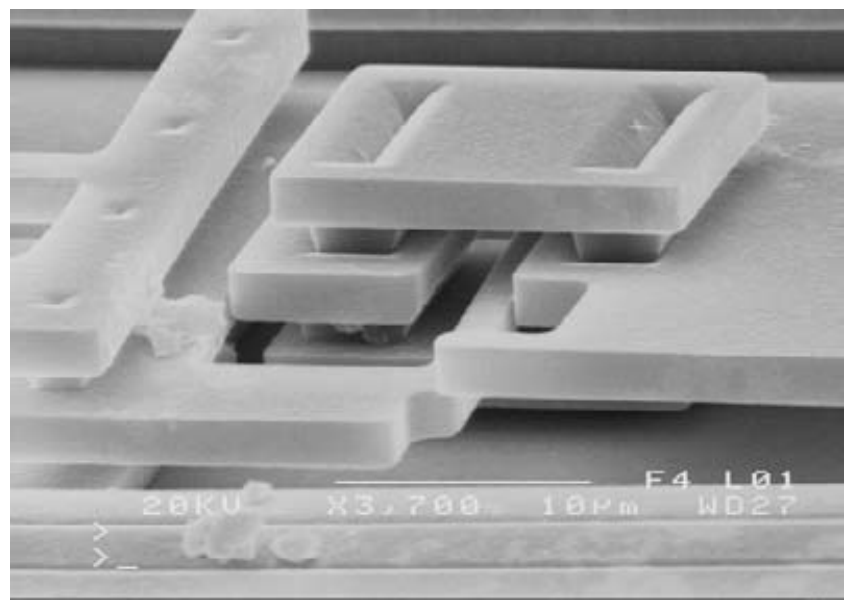

Fig. 13. SEM of a released hinge.

by the slider rails. This also constrains the axle of the hinge under the staple. Figure 12 shows an SEM of the slider hinge.

\section{III.6. Released hinge}

In SUMMiT $\mathrm{V}$ an additional hinge was used in some of the XYZM designs. Instead of using a scissor hinge to connect two released members, a release hinge was used. Because the SUMMiT V fabrication process uses 4 structural layers, a hinge could be fabricated that is similar to the substrate hinge. This hinge, instead of being anchored to the substrate, has a plate beneath the axle that prevents the axle from slipping out from beneath the bridge. An example is shown in Figure 13.

\section{RIGID-BODY DISPLACEMENT ANALYSES}

Although similar, the analyses for the XYZM and the OXYZM must be done separately because of differences in the motion of the device. In the motion of the XYZM, the surface of a sphere can be used to represent all possible positions of the leg. ${ }^{11,12}$ However, the addition of the offset in the OXYZM causes a deviation from this motion and an alternate method must be used to determine the platform position.
IV.1. XYZ Micromanipulator displacement analysis

The position of the platform for the XYZM is calculated by first determining the sphere of motion for each individual leg for an input displacement, $d_{i}$. After the spheres of motion are calculated for each leg, the system of equations is solved to find the intersection of these spheres. This intersection represents the position of the platform of the mechanism.

A loop-closure equation for the mechanism in the $\left(x_{i}, y_{i}, z_{i}\right)$ coordinate frame with respect to the origin can be expressed as

$$
\left[\begin{array}{c}
a \cos \theta_{1 i}+b \sin \theta_{3 i} \cos \left(\theta_{2 i}+\theta_{1 i}\right) \\
b \cos \theta_{3 i} \\
a \sin \theta_{1 i}+b \sin \theta_{3 i} \sin \left(\theta_{2 i}+\theta_{1 i}\right)
\end{array}\right]=\left[\begin{array}{c}
c_{x i} \\
c_{y i} \\
c_{z i}
\end{array}\right]
$$

where

$$
\left[\begin{array}{c}
c_{x i} \\
c_{y i} \\
c_{z i}
\end{array}\right]=\left[\begin{array}{ccc}
\cos \phi_{i} & \sin \phi_{i} & 0 \\
-\sin \phi_{i} & \cos \phi_{i} & 0 \\
0 & 0 & 1
\end{array}\right]\left[\begin{array}{c}
p_{x} \\
p_{y} \\
p_{z}
\end{array}\right]+\left[\begin{array}{c}
h-r \\
0 \\
0
\end{array}\right]
$$

in the $(x, y, z)$ coordinate frame and $i=1,2,3$, representing each individual leg of the device. In expanded form, Equations (1) and (2) each result in three systems of three equations, where each system represents the equations defining the position of point $C$ for one leg. $a$ represents the length of the lower section of the leg, and $b$ represents the link length of the parallel mechanism as shown in Figure 3. $\theta_{1 i}, \theta_{2 i}$, and $\theta_{3 i}$ represent the nine joint angles for $i=1,2,3$. $p_{x}, p_{y}$, and $p_{z}$ represent the $x, y$, and $z$ coordinates of point $P$ on the center of the platform with respect to the origin, $O$.

To determine the position of the platform given a certain displacement, Equations (1) and (2) are rearranged as

$$
\begin{aligned}
{\left[\begin{array}{c}
b \sin \theta_{3 i} \cos \left(\theta_{2 i}+\theta_{1 i}\right) \\
b \cos \theta_{3 i} \\
b \sin \left(\theta_{1 i}+\theta_{1 i}\right)
\end{array}\right]=} & {\left[\begin{array}{ccc}
\cos \phi_{i} & \sin \phi_{i} & 0 \\
-\sin \phi_{i} & \cos \phi_{i} & 0 \\
0 & 0 & 1
\end{array}\right]\left[\begin{array}{c}
p_{x} \\
p_{y} \\
p_{z}
\end{array}\right] } \\
& +\left[\begin{array}{c}
-a \cos \theta_{1 i}-r+h \\
0 \\
-a \sin \theta_{1 i}
\end{array}\right]
\end{aligned}
$$

Summing the squares for the three components in Equation (3) results in the equation for the sphere created by point $P$ during the full motion of leg $i$.

$$
\begin{aligned}
b^{2}= & p_{x}^{2}+p_{y}^{2}+p_{z}^{2} \\
& -2\left(p_{x} \cos \phi_{i}+p_{y} \sin \phi_{i}\right)\left(a \cos \theta_{i}+r-h\right) \\
& -2 p_{z} a \cos \theta_{1 i}+\left(a \cos \theta_{1 i}+r-h\right)^{2}+\left(a \sin \theta_{1 i}\right)^{2}
\end{aligned}
$$

To find the intersecting circle of the spheres created by leg 1 and leg $j$, Equation (4) for leg $i=1$ is subtracted from Equation (4) for $i=j$ resulting in

$$
e_{1 j} p_{x}+e_{2 j} p_{y}+e_{3 j} p_{z}+e_{4 j}=0
$$


where

$$
\begin{aligned}
e_{1 j}= & 2 \cos \phi_{j}\left(a \cos \theta_{1 j}+r-h\right) \\
& -2 \cos \phi_{1}\left(a \cos \theta_{11}+r-h\right) \\
e_{2 j}= & 2 \sin \phi_{j}\left(a \cos \theta_{1 j}+r-h\right) \\
& -2 \sin \phi_{1}\left(a \cos \theta_{11}+r-h\right) \\
e_{3 j}= & 2 a \sin \theta_{1 j}-2 a \sin \theta_{11} \\
e_{4 j}= & \left(a \cos \theta_{11}+r-h\right)^{2}+\left(a \sin \theta_{11}\right)^{2} \\
& -\left(a \cos \theta_{1 j}+r-h\right)^{2}-\left(a \sin \theta_{1 j}\right)^{2}
\end{aligned}
$$

and $j=2,3$.

Using Equation (5) for $j=2$ and $j=3$ results in a system of two equations for the intersecting circles of the spheres created by the motion of point $P$. The solutions for this system of equations results in the possible positions of the platform. Solving the equations for $p_{z}$ and $p_{y}$ in terms of $p_{x}$ and substituting them into Equation (4) for $i=1$, results in

$$
k_{0} p_{x}^{2}+k_{1} p_{x}+k_{2}=0
$$

where the coefficients for the quadratic equation in Equation (10) are

$$
\begin{aligned}
k_{0}= & 1+\frac{l_{1}^{2}}{l_{2}^{2}}+\frac{l_{4}^{2}}{l_{2}^{2}} \\
k_{1}= & \frac{2 l_{0} l_{1}}{l_{2}^{2}}+\frac{2 l_{3} l_{4}}{l_{2}^{2}}-2 l_{5} \cos \phi_{1} \\
& -\frac{2 l_{5} l_{1}}{l_{2}} \sin \phi_{1}-\frac{2 a l_{4}}{l_{2}} \sin \theta_{11} \\
k_{2}= & l_{5}^{2}-b^{2}+\frac{l_{0}^{2}}{l_{2}^{2}}+\frac{l_{3}^{2}}{l_{2}^{2}}+\left(a \sin \theta_{11}\right)^{2} \\
& -\frac{2 l_{0} l_{5}}{l_{2}} \sin \phi_{1}-\frac{2 a l_{3}}{l_{2}} \sin \theta_{11}
\end{aligned}
$$

where

$$
\begin{aligned}
& l_{0}=e_{32} e_{43}-e_{33} e_{42} \\
& l_{1}=e_{13} e_{32}-e_{12} e_{33} \\
& l_{2}=e_{22} e_{33}-e_{23} e_{32} \\
& l_{3}=e_{23} e_{42}-e_{22} e_{43} \\
& l_{4}=e_{12} e_{23}-e_{13} e_{22} \\
& l_{5}=a \cos \theta_{11}+r-h
\end{aligned}
$$

and $e_{12}, e_{13}, e_{22}, e_{23}, e_{33}, e_{42}$, and $e_{43}$ are defined in Equations (6) through (9).

After the value of $p_{x}$ is determined, the position of the center of the platform is found by substituting the value for $p_{x}$ into the system of two equations determined by Equation (5), and then solving for $p_{y}$ and $p_{z}$. The solution for this system of equations is

$$
\begin{aligned}
& p_{y}=\frac{l_{0}+l_{1} p_{x}}{l_{2}} \\
& p_{z}=\frac{l_{3}+l_{4} p_{x}}{l_{2}}
\end{aligned}
$$

There are three possible cases when solving for the position of the platform.

(i) If $k_{1}^{2}-4 k_{0} k_{2}>0$, then the spheres intersect at two locations and two solutions are possible.

(ii) If $k_{1}^{2}-4 k_{o} k_{2}=0$, then the three spheres intersect at a single point, resulting in one real solution.

(iii) If $k_{1}^{2}-4 k_{o} k_{2}<0$, then the three spheres do not have an intersection point and there is no solution for the position.

The above equations relate the displacement of the platform to the input angles, $\theta_{1 i}$. This angle can be related to the input displacement at the slider, $d_{i}$, by the following equation:

$$
\theta_{1 i}=a \cos \left(\frac{\left(a+r_{2}-d_{i}\right)^{2}+a^{2}-r_{2}^{2}}{2 a\left(a+r_{2}-d_{i}\right)}\right)
$$

for $i=1,2,3$.

After the position is known and $c_{x i}, c_{y i}$, and $c_{z i}$ are determined using (2), the following equations, obtained from (i), can be used to determine angles $\theta_{2 i}$ and $\theta_{3 i}$ :

$$
\begin{gathered}
\theta_{3 i}=a \cos \left(\frac{c_{y i}}{b}\right) \\
\theta_{2 i}=a \cos \left(\frac{c_{x i}^{2}+c_{y i}^{2}+c_{z i}^{2}-a^{2}-b^{2}}{2 a b \sin \theta_{3 i}}\right)
\end{gathered}
$$

The necessary input displacement for each leg can then be calculated using Equation (22).

\section{IV.2. Offset $X Y Z$ micromanipulator analysis}

The displacement analysis for the OXYZM is similar to the $\mathrm{XYZM}$, however, because of the offsets the solution is no longer closed form. Therefore, to solve for the position of the device, an iterative method must be used.

A loop-closure equation for the mechanism in the $\left(x_{i}, y_{i}, z_{i}\right)$ coordinate frame including offsets, $f_{1}$ and $f_{2}$, can be expressed as:

$$
\begin{aligned}
& {\left[\begin{array}{c}
a \cos \theta_{1 i}+b \sin \theta_{3 i} \cos \left(\theta_{2 i}+\theta_{1 i}\right)+\left(f_{1}+f_{2}\right) \cos \left(\theta_{2 i}+\theta_{1 i}\right) \\
b \cos \theta_{3 i} \\
a \sin \theta_{1 i}+b_{o} \sin \theta_{3 i} \sin \left(\theta_{2 i}+\theta_{1 i}\right)+\left(f_{1}+f_{2}\right) \sin \left(\theta_{2 i}+\theta_{1 i}\right)
\end{array}\right]} \\
& \quad=\left[\begin{array}{c}
c_{x i} \\
c_{y i} \\
c_{z i}
\end{array}\right]
\end{aligned}
$$

where

$$
\left[\begin{array}{c}
c_{x i} \\
c_{y i} \\
c_{z i}
\end{array}\right]=\left[\begin{array}{ccc}
\cos \phi_{i} & \sin \phi_{i} & 0 \\
-\sin \phi_{i} & \cos \phi_{i} & 0 \\
0 & 0 & 1
\end{array}\right]\left[\begin{array}{c}
p_{x} \\
p_{y} \\
p_{z}
\end{array}\right]+\left[\begin{array}{c}
h-r \\
0 \\
0
\end{array}\right]
$$


Table I. Rigid-body XYZ Micropositioning Mechanisms Link Lenghs for Designs Fabricated using the MUMPs Process.

\begin{tabular}{lc}
\hline Link & Length \\
\hline$a$ & $65 \mu \mathrm{m}$ \\
$b$ & $108 \mu \mathrm{m}$ \\
$h$ & $110 \mu \mathrm{m}$ \\
$r$ & $365 \mu \mathrm{m}$ \\
$r_{2}$ & $300 \mu \mathrm{m}$ \\
$f_{1}$ & 85 \\
$f_{2}$ & 120 \\
\hline
\end{tabular}

Table II. Rigid-body XYZ Micromanipulator Link Lengths in SUMMi T V.

\begin{tabular}{lrr}
\hline Link & Design 1 & Design 2 \\
\hline$a$ & $200 \mu \mathrm{m}$ & $125 \mu \mathrm{m}$ \\
$b$ & $130 \mu \mathrm{m}$ & $188 \mu \mathrm{m}$ \\
$h$ & $95 \mu \mathrm{m}$ & $118 \mu \mathrm{m}$ \\
$r$ & $95 \mu \mathrm{m}$ & $170 \mu \mathrm{m}$ \\
$r_{2}$ & $125 \mu \mathrm{m}$ & $165 \mu \mathrm{m}$ \\
$f_{1}$ & $30 \mu \mathrm{m}$ & $0 \mu \mathrm{m}$ \\
$f_{2}$ & $30 \mu \mathrm{m}$ & $0 \mu \mathrm{m}$ \\
\hline
\end{tabular}

Equation (25) results in a system of nonlinear equations requiring iteration to obtain a solution for the platform position. Therefore the analysis for the XYZM should be performed first to determine the initial values to be used in the iteration for the OXYZM analysis. In using the XYZM analysis as the starting point, the sum of the offsets $\left(f_{1}+f_{2}\right)$ should be added to the link length of the parallelogram mechanism, $b$. After the initial analysis is complete, substitute the dependent angles, $\theta_{2 i}$ and $\theta_{3 i}$, and the position coordinates, $p_{x}, p_{y}$, and $p_{z}$, into Equation (25) and solve the system of equations determine the final position of the OXYZM. Perturbation theory provides the justification of using the results from the XYZM displacement analysis as a starting point.

Table I lists the values used in the link lengths of the rigidbody OXYZM fabricated using the MUMPs process. Two designs (XYZM and an OXYZM) of the rigid-body XYZM were fabricated in SUMMiT V and values for the link lengths are listed in Table II.

\section{IV.3. Perturbation theory}

Perturbation theory is a collection of iterative methods used for the analysis of nonlinear equations. ${ }^{21}$ In perturbation theory, a parameter, $\varepsilon$, is identified such that when $\varepsilon=0$ equations are solved in closed form.

In the case of the OXYZM, the offsets can be represented by this small parameter such that

$$
\varepsilon=f_{1}+f_{2}
$$

when $\varepsilon=0$, the offset XYZM analysis is identical to the $\mathrm{XYZM}$ analysis and becomes a linear system.
Introducing $\varepsilon$ into Equation (25) results in:

$$
\left[\begin{array}{c}
c_{x i} \\
c_{y i} \\
c_{z i}
\end{array}\right]=\left[\begin{array}{c}
a \cos \theta_{1 i}+b \sin \theta_{3 i} \cos \left(\theta_{2 i}+\theta_{1 i}\right)+\varepsilon \cos \left(\theta_{2 i}+\theta_{1 i}\right) \\
b \cos \theta_{3 i} \\
a \sin \theta_{1 i}+b \sin \theta_{3 i} \sin \left(\theta_{2 i}+\theta_{1 i}\right)+\varepsilon \sin \left(\theta_{2 i}+\theta_{1 i}\right)
\end{array}\right]
$$

Figure 6 shows that

$$
r+a=h+b \sin \theta_{3 i}+\left(f_{1}+f_{2}\right)
$$

Therefore, $r+a$ is used to nondimensionalize $c$ and can be represented by

$\left[\begin{array}{c}\hat{c_{x i}} \\ \hat{c_{y i}} \\ \hat{c_{z i}}\end{array}\right]=\left[\begin{array}{c}\hat{a} \cos \theta_{1 i}+\hat{b} \sin \theta_{3 i} \cos \left(\theta_{2 i}+\theta_{1 i}\right)+\varepsilon \cos \left(\theta_{2 i}+\theta_{1 i}\right) \\ \hat{b} \cos \theta_{3 i} \\ \hat{a} \sin \theta_{1 i}+\hat{b} \sin \theta_{3 i} \sin \left(\theta_{2 i}+\theta_{1 i}\right)+\varepsilon \sin \left(\theta_{2 i}+\theta_{1 i}\right)\end{array}\right]$

and shows that $\varepsilon$ is very small in comparison to the other values in the system of equations.

The following equations are used to approximate the solutions for the system of nonlinear equations using perturbation methods

$$
\begin{aligned}
\hat{c_{i}} & =c_{0 i}+\varepsilon c_{1 i}+\varepsilon^{2} c_{2 i}+\cdots \\
\hat{P} & =P_{0}+\varepsilon P_{1}+\varepsilon^{2} P_{2}+\cdots \\
\theta_{2 i} & =\theta_{2 i 0}+\varepsilon \theta_{2 i 1}+\varepsilon^{2} \theta_{2 i 2}+\cdots \\
\theta_{3 i} & =\theta_{3 i 0}+\varepsilon \theta_{3 i 1}+\varepsilon^{2} \theta_{3 i 2}+\cdots
\end{aligned}
$$

The zeroth order terms $\left(c_{0 i}, P_{0}, \theta_{2 i 0}\right.$, and $\left.\theta_{3 i 0}\right)$ are obtained by solving for the system with $\varepsilon=0$. The first order terms may be calculated to ensure that each value is a converging series. Therefore, when $\varepsilon$ is sufficiently small in comparison to the dimension values being sought, a good approximation can be determined by solving for the system when $\varepsilon=0$. For this reason, the $X Y Z$ analysis is used to determine the initial values for use in the iterations of the OXYZM analysis, thus allowing the nonlinear system to converge to a solution more quickly.

\section{IV.4. Rigid-body XYZM testing}

The rigid-body XYZM designs could be actuated with microprobes using several different input displacements. With an input displacement of 45 micrometers on each leg, the XYZM achieved a predicted out-of-plane displacement of 188 micrometers. The offset XYZM achieved a predicted displacement of 205 micrometers with 45 micrometer input displacements. These experimental results compare favorably to the predicted results listed in Table III. Experimentally measured output forces are not available.

The clearances in the pin joints of the parallelogram mechanism caused two challenges. First, the clearances are large enough that the associated backlash can cause significant deviation from the ideal motion. Second, binding can occur at these joints. These negative effects can be mitigated by replacing the joint function with compliant 
Table III. XYZM output displacement components of platform, $\mathrm{cx}, \mathrm{cy}$, and cz, given slider input displacements, $\mathrm{d} 1$, $\mathrm{d} 2$, and $\mathrm{d} 3$.

\begin{tabular}{lcrrrc}
\hline $\mathrm{d}_{1}$ & $\mathrm{~d}_{2}$ & $\mathrm{~d}_{3}$ & \multicolumn{1}{c}{$\mathrm{c}_{\mathrm{X}}$} & \multicolumn{1}{c}{$\mathrm{c}_{\mathrm{y}}$} & $\mathrm{c}_{\mathrm{Z}}$ \\
\hline $30 \mu \mathrm{m}$ & $30 \mu \mathrm{m}$ & $30 \mu \mathrm{m}$ & $0 \mu \mathrm{m}$ & $0 \mu \mathrm{m}$ & $162 \mu \mathrm{m}$ \\
$30 \mu \mathrm{m}$ & $40 \mu \mathrm{m}$ & $50 \mu \mathrm{m}$ & $12 \mu \mathrm{m}$ & $7 \mu \mathrm{m}$ & $179 \mu \mathrm{m}$ \\
$20 \mu \mathrm{m}$ & $30 \mu \mathrm{m}$ & $50 \mu \mathrm{m}$ & $16 \mu \mathrm{m}$ & $13 \mu \mathrm{m}$ & $165 \mu \mathrm{m}$ \\
$30 \mu \mathrm{m}$ & $35 \mu \mathrm{m}$ & $40 \mu \mathrm{m}$ & $6 \mu \mathrm{m}$ & $3 \mu \mathrm{m}$ & $171 \mu \mathrm{m}$ \\
$10 \mu \mathrm{m}$ & $20 \mu \mathrm{m}$ & $50 \mu \mathrm{m}$ & $20 \mu \mathrm{m}$ & $19 \mu \mathrm{m}$ & $148 \mu \mathrm{m}$ \\
$45 \mu \mathrm{m}$ & $45 \mu \mathrm{m}$ & $45 \mu \mathrm{m}$ & $0 \mu \mathrm{m}$ & $0 \mu \mathrm{m}$ & $188 \mu \mathrm{m}$ \\
\hline
\end{tabular}

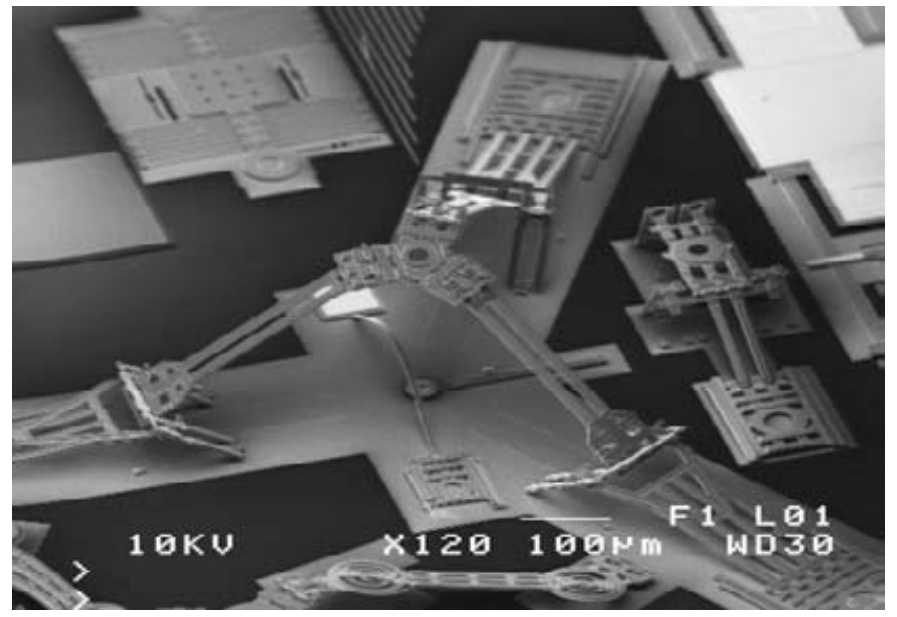

Fig. 14. SEM of Compliant XYZ Micromanipulator fabricated using the MUMPs process.

mechanisms. The design of a compliant XYZM is described next.

\section{COMPLIANT XYZ MICROMANIPULATOR}

In the compliant XYZM, the rigid-body parallel mechanism found in the upper section of the leg is replaced with a compliant parallel mechanism. ${ }^{22}$ Compliant versions of this mechanism were fabricated using both MUMPs and SUMMiT V. An SEM of a compliant XYZM is shown in Figure 14.

\section{V.1. Compliant mechanisms}

Compliant mechanisms gain some or all of their motion from the deflection of flexible segments. Compliant mechanism can be either fully-compliant, where the mechanism gains all motion from this deflection, or partially compliant, where the mechanism gains part of its motion from deflection.

The advantages of compliant mechanisms over traditional rigid-body mechanisms are that they are more wear resistant due to the elimination of rubbing parts. Compliant mechanisms are especially advantageous in micropositioning and nanopositioning applications because clearance and backlash in the pin joints are eliminated, resulting in a mechanism that is more precise, predictable, and reliable.

The main disadvantage of compliant mechanisms is the difficulty of analysis due to nonlinearities in the geometry of mechanisms during large deflections. However, the pseudo- rigid-body model, ${ }^{22}$ has provided a simple method to analyze these mechanisms by modeling the flexible segments as rigidbody links with torsional springs. This then allows traditional kinematic methods to be used for analysis.

\section{V.2. Pseudo-rigid-body model}

In the Compliant XYZM, the parallelogram mechanism in the upper section of the leg was replaced with a compliant parallel mechanism and was modelled as a rigid-body parallel mechanism with torsional springs. In the pseudo-rigid bodymodel, the key is to determine the location of the pin joint and the values of the torsional spring constants. The pseudorigid-body model introduces the following variables into the XYZM displacement analysis:

$\gamma=$ characteristic radius factor

$l=$ length of compliant link

$\Theta=$ pseudo-rigid-body angle

The characteristic radius factor, $\gamma$ is determined from the orientation of the input force. In the case with the XYZM, the input force on the parallel guiding mechanism is assumed to be horizontal and the value is a constant 0.85 . The characteristic radius, $\gamma l$, represents the length of the pseudorigid-body link. $\Theta$ represents the angle between the pseudorigid-body link and its undeflected position. Figure 15a shows the schematic of the leg with the compliant mechanism in the upper section. Figure 15b shows the schematic of the pseudorigid body model of a single leg. Table IV lists the link lengths of the MUMPs and SUMMiT V designs.

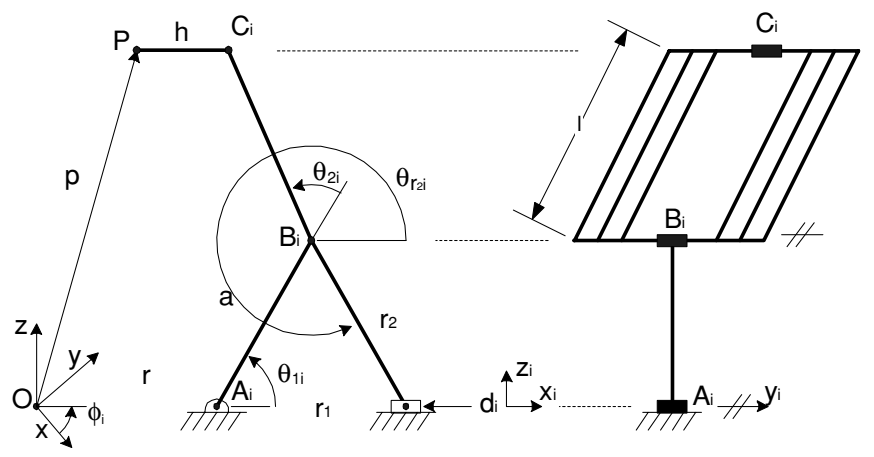

a)

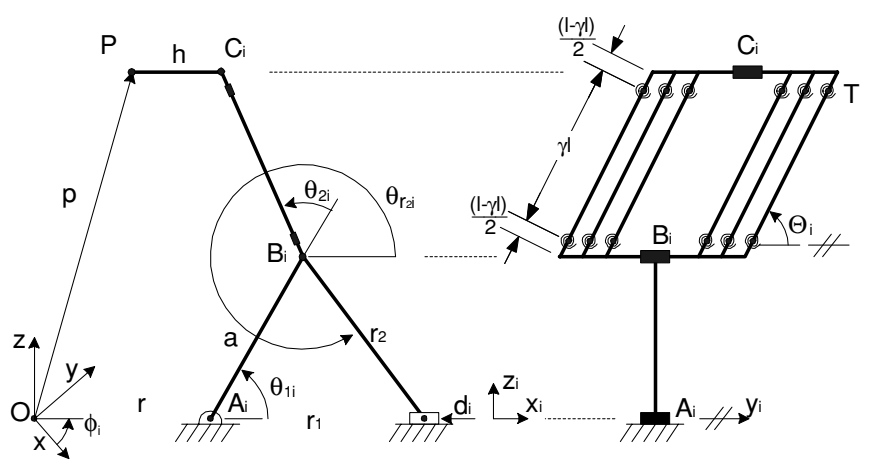

b)

Fig. 15. Schematic of single leg of Compliant XYZ Micropositioning Mechanism. a) Compliant leg b) Pseudo-Rigid Body Model of Leg. 
Table IV. Link lengths for compliant XYZ Micropositioning Mechanism fabricated using the MUMPs fabrication process.

\begin{tabular}{lrc}
\hline Link & MUMPs & SUMMiT V \\
\hline$a$ & $100 \mu \mathrm{m}$ & $300 \mu \mathrm{m}$ \\
$l$ & $300 \mu \mathrm{m}$ & $250 \mu \mathrm{m}$ \\
$h$ & $80 \mu \mathrm{m}$ & $100 \mu \mathrm{m}$ \\
$r$ & $250 \mu \mathrm{m}$ & $100 \mu \mathrm{m}$ \\
$r_{2}$ & $300 \mu \mathrm{m}$ & $180 \mu \mathrm{m}$ \\
\hline
\end{tabular}

\section{V.3. Compliant displacement analysis and testing}

The equations for the displacement analysis for the compliant version of the XYZM are similar to the analysis for the offset rigid-body XYZM. However, to take into account the effects the compliant parallel-guiding mechanism, link $b$ is replaced with $\gamma l,\left(f_{1}+f_{2}\right)$ is replaced by $(l-\gamma l)$, and the angle $\theta_{3 i}$ is replaced by $\Theta_{i}$. This results in the following loopclosure equation for the compliant XYZM in the $\left(x_{i}, y_{i}, z_{i}\right)$ coordinate frame:

$$
\begin{aligned}
& {\left[\begin{array}{c}
a \cos \theta_{1 i}+\gamma l \sin \Theta_{i} \cos \theta_{2 i}+(l-l \gamma) \cos \left(\theta_{2 i}+\theta_{1 i}\right) \\
\gamma l \cos \Theta_{i} \\
a \sin \theta_{1 i}+\gamma l \sin \Theta_{i} \sin \theta_{2 i}+(l-l \gamma) \sin \left(\theta_{2 i}+\theta_{1 i}\right)
\end{array}\right]} \\
& \quad=\left[\begin{array}{c}
c_{x i} \\
c_{y i} \\
c_{z i}
\end{array}\right]
\end{aligned}
$$

where the pseudo-rigid body angle is:

$$
\Theta=\left(\theta_{3 i}-\theta_{3 i o}\right)
$$

$\theta_{3 i}$ represents the angle of the compliant segment in its deflected position and $\theta_{3 i o}$ represents the angle of the compliant segment in its undeflected position. The remainder of the compliant XYZM analysis follows that of the rigidbody OXYZM with these substitutions throughout the model. Like the OXYZM, the compliant XYZM also results in a system of nonlinear equations. The XYZM analysis model with $b=\gamma l$ provides the initial guess for the iterative solution of Equation (35).

In the testing of the compliant XYZM, micro probes were also used at each of the three input positions to displace the sliders. The compliant XYZM achieved a predicted displacements of 262 micrometers using 45 micrometer displacements at each slider. The compliant XYZM did not experience the binding that was evident in the rigidbody mechanism and could consistently be actuated without binding or failure into the orthoplanar position when input forces were linear with the slider of the device. Forcedisplacement relationships may also be derived for the mechanisms, as described in reference [23].

\section{CONCLUSION}

Several XYZ Micromanipulators have been fabricated and tested successfully using two surface micromachining processes and found to achieve out-of-plane displacement with three linear inputs while maintaining a horizontal position of the platform throughout its motion. The compliant XYZM was more consistent in its position by the elimination of backlash in the rigid-body pin-joints and was more easily actuated.

The XYZ Micromanipulator has applications in moving and positioning microcomponents such as mirrors, lenses, and gratings. It can position micro-components in the $x, y$, and $z$ directions by using three independent linear inputs and has achieved out-of-plane displacements greater than 250 micrometers. The XYZM can be used in applications where significant out-of-plane displacements of microcomponents are necessary and/or varied positions in the $x, y$, and $z$ directions are desired.

\section{Acknowledgements}

The support of the National Science Foundation through grant CMS-9978737 is gratefully acknowledged. The assistance Jon Wittwer is also greatly appreciated.

\section{References}

1. E. Ollier, C. Charbrol, T. Enot, P. Brunet-Manquat, J. Margail and P. Mottier, " $1 \times 8$ Micro-Mechanical Switches based on moving waveguides for Optical Fiber Network Switching," Proceedings 2000 IEEE/LEOS International Conference on Optical MEMS, Kauai, HI (Aug., 2000) pp. 39-40.

2. M. C. Wu, "Micromachining for Optical and Optoelectronic Systems," Proceedings of the IEEE (Nov., 1987) Vol. 85, No 11, pp. 1833-1856.

3. C. R. King, L. Y. Lin and M. C. Wu, "Out-of-Plane Refractive Microlens Fabricated by Surface Micromachining," IEEE Photonics Technology Letters 8, No 10, 1349-1359 (Oct., 1996).

4. M. E. Motamedi, M. C. Wu and K. S. J. Pister, "Micro-opto-electro-mechanical devices and on-chip optical processing," Optical Engineering 36, No 5, 1282-1297 (May, 1997).

5. L. Y. Lin, S. S. Lee, K. S. J. Pister and M. C. Wu, "MicroMachined Three-Dimensional Micro-Optics for Integrated Free-Space Optical System," IEEE Photonics Technology Letters 6, No 12, 1445-1447 (Dec., 1994).

6. C. Marxer, C. Thio, M. Gretillat and N. de Rooij,"Vertical Mirrors Fabricated by Deep Reactive Ion Etching for Fiber-optic Switching Applications," Journal of Microelectromechanical Systems 6, No 3, 277-285 (Sept., 1997).

7. H. Nguyen, J. G. Su, H. Toshiyoshi and M. C. Wu, "Device Transplant of Optical MEMS of Out of Plane Beam Steering," Proceedings of the Fourteenth IEEE International Conference on Micro Electro Mechanical Systems (2001) pp. 325- 328.

8. Y. Lin, E. L. Goldstein, L. M. Lunardi and R. W. Tkach, "1999 Micromachined Optical-Switching Technologies for WDM networks," Proceedings of the 1999 IEEE/LEOS Summer Topical Meeting on Nanostructures and Quantum Dots (July 26-July 27, 1999) pp. 57-58.

9. R. T. Chen, H. Nguyen and M. C. Wu, "A High Speed LowVoltage Stress-Induced Micromachined $2 \times 2$ Optical Switch," IEEE Photonics Technology Letters 11, No 11, 1396-1398 (Nov., 1999).

10. S. S. Lee, L. Y. Lin and M. C. Wu, "Surface Micromachined free-space micro-optical systems containing three-dimensional microgratings," Applied Physics Letters 67, No 15, 2135-2137 (9 Oct., 1995)

11. L. W. Tsai and R. Stamper, "A Parallel Manipulator with Only Translational Degrees of Freedom," '96-DETC-MECH-1152, 
ASME 1996 Design Engineering Technical Conferences, Irvine, CA (1996) pp. 1-8.

12. L. W. Tsai, Robot Analysis, The Mechanics of Serial and Parallel Manipulators (John Wiley and Sons, Inc. New York, 1999).

13. D. Kim and W. K. Chung, "Kinematic Condition Analysis of Three-DOF Pure Translational Parallel Manipulators," Journal of Mechanical Design 125, 323-331 (June, 2003).

14. J. J. Parise, L. L. Howell and S. P. Magleby, "Ortho-Planar Mechanisms," Proceedings of the ASME Design Engineering Technical Conferences and Computers and Information in Engineering Conference, Baltimore, Maryland (Sept. 10-13, 2000) pp. 1-8.

15. D. A. Koester, A. Cowen, R. Mahadevan, M. Stonefield and B. Hardy, PolyMUMPS Design Handbook, Revision 10.0 (MEMSCAP, 2003).

16. J. J. Sniegowski and M. P. de Boer, "IC-compatible Polysilicon Surface Micromachining," Ann. Rev. Mater. Sci. 30, 299-333 (2000).

17. D. Clements, L. L. Howell, N. Masters and B. Weight, "Floating Pin Joints Fabricated from Two Layers of Polysilicon at the Micro Level," Tenth World Conference on the Theory of
Machines and Mechanisms, Oulu, Finland (June 20-24, 1999) Vol. 2, pp. 874-879.

18. R. Yeh, E. J. J. Kruglick and K. S. J. Pister, "SurfaceMicromachined Components for Articulated Microrobots," Journal of Microelectromechanical Systems 5, No 1, 10-17 (March, 1996).

19. A. Friedberger and R. S. Muller, "Improved SurfaceMicromachined Hinges for Fold-Out Structures," Journal of Microelectromechanical Systems 7, No 3, 315-319 (Sept., 1998).

20. Y. W. Yi and C. Liu, "Magnetic Actuation of Hinged Microstructures," Journal of Microelectromechanical Systems 8, No 1, 10-17 (March, 1999).

21. C. M. Bender and S. A. Orszag, Advanced Mathematical Methods for Scientists and Engineers (McGraw-Hill Book Company, United States of America, 1978).

22. L. L. Howell, Compliant Mechanisms (John Wiley and Sons, Inc., New York, 2001).

23. K. A. Jensen, C. P. Lusk and L. L. Howell, "Force Relationships for an XYZ Micromanipulator with Three Translational Degrees of Freedom," Proceedings of the 2004 ASME Mechanisms and Robotics Conference, DETC 200457028(2004) pp. 1-8. 